\title{
Invited Comment on G. Bassotti and E. Battaglia: Postoperative colonic motility after transverse loop colostomy: a further tassel in the mosaic
}

\author{
G. Bassotti · E. Battaglia
}

Received: 25 June 2014/ Accepted: 25 June 2014/ Published online: 19 July 2014

(C) Springer-Verlag Italia Srl 2014

It is generally agreed that surgical procedures involving the gastrointestinal tract may, to a greater or lesser extent, influence or disrupt its motor activity by removing parts of the gut or creating anastomoses [1]. However, notwithstanding this almost universal belief, it is interesting and noteworthy that there are only a handful of studies in humans that investigated this aspect of intestinal function and evaluated the motility features in the postoperative colon [1]. This fact is even more surprising since manometric recordings for prolonged periods of time $(24 \mathrm{~h})$ both in healthy volunteers [2] and in patients with different pathological conditions [3, 4] have provided us with a relatively good knowledge of the motor behavior of the human colon.

Indeed, manometric evaluations in the operated colon are quite rare in the literature, and most of the available data are limited to left hemicolectomy (which, in contrast to major intestinal surgery without anastomosis, has a major inhibitory effect on distal colonic motility), right hemicolectomy (which, even in the long term, seems to modify only slightly the colonic motor function) [5], and anterior resection of the rectum (followed by anterior resection syndrome which consists of a wide spectrum of symptoms caused by motility disorders [6]), characterized by a huge increase of the colonic propulsive activity [7], the so-called high-amplitude propagated contractions (HAPC, the manometric equivalent of radiologically

G. Bassotti $(\bowtie)$

Gastroenterology Section, Department of Medicine, University of Perugia Medical School, Piazza Lucio Severi, 1, 06132 San Sisto (Perugia), Italy

e-mail: gabassot@tin.it

E. Battaglia

Gastroenterology Unit, Cardinal Massaia Hospital, Asti, Italy described mass movements) [8]. Thus, studies addressing the various aspects of colonic motor activity in various postoperative settings are particularly welcome.

In this issue, Pucciani and colleagues report for the first time a group of patients who had undergone loop colostomy, in whom manometric recordings were simultaneously obtained before and after a meal in both the afferent and efferent loop [9]. These recordings showed that colonic motility in these patients, similar to that in healthy volunteers $[2,7]$, was characterized by alternating periods of motor quiescence and apparently random contractions, and a significant correlation was found between HAPC and bowel evacuation. In about $17 \%$ of cases, no such activity was recorded; but this is not surprising, since liquid stools may be also propelled by low-amplitude propagated waves [8]. In addition, the remaining colon maintained a valid colonic motor response to food intake [10], with a significant increase in motility in both loops after a $950 \mathrm{kcal}$ meal. The colon distal to the anastomosis displayed significantly lower motor activity compared to that recorded in the proximal loop. Interestingly, both highand low-amplitude propagated activity could sometimes be recorded, and most of these contractions spread in an arboreal direction even though in three instances retrograde contractions were documented. However, retrograde HAPC can be documented in healthy subjects although not frequently [8]. The authors conclude that transverse loop colostomy does not disrupt the various patterns of colonic motility, and that with respect to this surgical approach, the only benefits to be expected are those related to exclusion of the fecal stream, whereas one cannot foresee any favorable effect related to decreased motor activity. Some points are worth commenting on.

Firstly, although the group of patients investigated was relatively small, it was also relatively homogeneous from 
both a clinical, laboratory, and instrumental point of view, strengthening the authors' results and improving the clinical value of their observations. Unfortunately, some clinical information, such as the presence of abdominal pain, distention, or bloating that may have been useful to better interpret the manometric data, is not provided.

Secondly, the recording time (although consistent with standard recommendations) was relatively short. This may have some importance in the colon, in which the various motility patterns (motor quiescence, regular and irregular activity, response to a meal) may be fully recorded only with prolonged $(24 \mathrm{~h})$ recordings [8]. Thus, probably due to the limited recording time, the authors were unable to document any regular contractile activity, such as that at 3 or 6 cycles per minute, and activity that may be related to symptoms, particularly abdominal pain, in some subgroups of patients (even though the authors were careful to exclude patients with concomitant symptoms related to an irritable bowel syndrome) [11].

The above points indicate how difficult it can be to evaluate patients postoperatively, and studies such as that of Pucciani and colleagues are welcome, and further research on this topic should be encouraged since it will most likely contribute to a better understanding, and management, of patients undergoing surgical procedures on the large bowel. For instance, as regards the present study, it remains to be established whether after stoma closure the almost normal motor behavior of the colon will persist or whether reestablishing intestinal continuity after (temporary) anatomic resection of the colon and interruption of fecal flow will influence the behavior of the large bowel and induce symptoms, possibly due to distention of the intestinal wall by the arrival of intestinal contents [12]. Thus, follow-up studies are needed and we hope that the authors will also investigate these patients after stoma closure, in order to give us the complete picture of colonic motor activity after loop colostomy, and to add further tesserae to the mosaic of overall postoperative colonic motility.
Conflict of interest None.

\section{References}

1. Bassotti G, Villanacci V, Battaglia E, Maurer CA (2014) In Quigley EMM, Hongo M, Fukudo S, eds. Functional and GI motility disorders. Specific clinical context—part 4: the postoperative patient. Front Gastrointest Res 33:163-169

2. Bassotti G, Imbimbo BP, Gaburri M, Daniotti S (1987) Transverse and sigmoid colon motility in healthy humans: effects of eating and cimetropium bromide. Digestion 37:59-64

3. Bassotti G, Chistolini F, Marinozzi G, Morelli A (2003) Abnormal colonic propagated activity in patients with slow transit constipation and constipation-predominant irritable bowel syndrome. Digestion 68:178-183

4. Bassotti G, Chistolini F, Nzepa FS, Morelli A (2003) Colonic propulsive impairment in intractable slow-transit constipation. Arch Surg 138:1302-1304

5. Baxter NN, Pemberton JH (2002) Postoperative motility disorders. In: Schuster MM, Crowell MD, Koch KL (eds) Schuster Atlas of gastrointestinal motility, 2nd edn. BC Decker, Hamilton, pp 353-370

6. Bryant CL, Lunniss P, Knowles CH, Thaha MA, Chan CLH (2012) Anterior resection syndrome. Lancet Oncol 13:e403-e408

7. Bassotti G, de Roberto G, Chistolini F, Morelli A, Pucciani F (2005) Case report: colonic manometry reveals abnormal propulsive behaviour after anterior resection of the rectum. Dig Liver Dis $37: 124-128$

8. Bassotti G, Iantorno G, Fiorella S, Bustos-Fernandez L, Bilder CR (1999) Colonic motility in man: features in normal subjects and in patients with chronic idiopathic constipation. Am J Gastroenterol 94:1760-1770

9. Pucciani F, Ringressi MN, Maltinti G, Bechi P (2014) Transverse loop colostomy and colonic motility. Tech Coloproctol. doi:10. 1007/s10151-014-1173-5

10. Bassotti G, Morelli A, Whitehead WE (1992) Abnormal rectosigmoid myoelectric response to eating in patients with severe idiopathic constipation (slow-transit type). Dis Colon Rectum 35:753-756

11. Bassotti G, Sietchiping-Nzepa F, De Roberto G, Chistolini F, Morelli A (2004) Colonic regular contractile frequency patterns in irritable bowel syndrome: the 'spastic colon' revisited. Eur J Gastroenterol Hepatol 16:613-617

12. Bassotti G, Gaburri M, Imbimbo BP, Morelli A, Whitehead WE (1994) Distension-stimulated propagated contractions in human colon. Dig Dis Sci 39:1955-1960 